\title{
Association of PIP5K2A gene polymorphisms with the effectiveness of the therapy of current depressive episode
}

\author{
N.M. Vyalova*, G.G. Simutkin, S.A. Ivanova \\ Mental Health Research Institute of the Federal State Budget Scientific Institution, Tomsk, Russia \\ *e-mail: Natarakitina@yandex.ru
}

Key words: PIP5K2A kinase, depressive disorders, genotyping, polymorphic variants

Motivation and Aim: The search for genetic markers associated with the development and course of depressive disorders, will allow developing effective methods of diagnosis and treatment of the disease. Modern studies indicate the involvement of PIP5K2A kinase in the pathogenesis of depressive disorders and schizophrenia spectrum disorders. The mechanisms underlying the therapeutic effect in chronic lithium treatment for bipolar affective disorders, is associated with differential expression of genes associated with phosphoinositide metabolism, including PIP5K2A. The aim of our study was to assess the effectiveness of therapy current depressive episode based on the definition of polymorphic variants rs10828317 and rs 10430590 PIP5K2A gene, previously studied by us both associated with depressive disorders. Methods and Algorithms: We examined 306 patients with depressive disorders were diagnosed as having current depressive episodes of varying severity within F31-F32, aged 20 to 60 years who were treated at the Department of Affective States clinics of Mental Health Research Institute. Assessment of the severity of the current depressive episode was performed using the scales SIGH-SAD, CGI-S, CGI-I, allowing to assess the severity of the disease before, on the 14th and 28th days of therapy. The control group consisted of 147 mentally and somatically healthy donors Russian population of the Siberian region of Tomsk and Tomsk region) aged 20 to 60 years. As the material for the study was used venous blood. DNA was isolated by standard phenol-chloroform micro technique. Genotyping for polymorphic variants $P I P 5 K 2 A$ gene was performed by polymerase-chain reaction (PCR) in real time using fluorescence on the amplifier "Step One Plus" company Applied Biosystems (USA). Statistical processing of results was performed using the program SPSS 20.0. Results: For studying the link between severity of depression and the assessment of the effectiveness of the therapy with the PIP5K2A gene polymorphism and the study of dependence of average aggregate scores in CGI-I, CGI-S and SIGH-SAD from the studied genotypes in patients with depressive disorders before therapy and on the 14th and on 28th day of treatment. Before the treatment, all patients had significantly high scores on the scale SIGH-SAD, CGI-I, CGI-S, and on the 14th and 28th days of therapy was observed significant decrease in scores on all scales investigated, which indicates an improvement in the clinical condition of patients. Our results revealed the association of the polymorphic variants rs10828317 PIP5K2A gene with the score for a typical depressive symptoms on a scale SIGH-SAD to initiation of therapy and with the score on a scale CGI-S at day 28 of therapy, and rs10430590 PIP5K2A gene shows a statistically significant association with the score on a scale CGI-S at day 28 of therapy.

Conclusion: Association of polymorphic variants of the gene PIP5K2A with the amount of points for a typical depressive symptoms on a scale SIGH-SAD with initiation of therapy and with an average amount of points on a scale CGI-S at day 28 of therapy on the background of clinical improvement proves the involvement of PIP5K2A kinases in the mechanisms underlying the therapeutic effect of antidepressants.

Acknowledgements: Supported by the RFBR No. 17-29-0205. 